Preprint typeset in JHEP style. - HYPER VERSION

SWAT-350

\title{
Exact Superpotentials from Matrix Models
}

\author{
Nick Dorey, Timothy J. Hollowood, S. Prem Kumar and Annamaria Sinkovics \\ Department of Physics, University of Wales Swansea, Swansea, SA2 8PP, UK \\ E-mail: t.hollowood@swan.ac.uk, n.dorey@swan.ac.uk \\ s.p.kumar@swan.ac.uk, a.sinkovics@swan.ac.uk
}

\begin{abstract}
Dijkgraaf and Vafa (DV) have conjectured that the exact superpotential for a large class of $\mathcal{N}=1$ SUSY gauge theories can be extracted from the planar limit of a certain holomorphic matrix integral. We test their proposal against existing knowledge for a family of deformations of $\mathcal{N}=4$ SUSY Yang-Mills theory involving an arbitrary polynomial superpotential for one of the three adjoint chiral superfields. Specifically, we compare the DV prediction for these models with earlier results based on the connection between SUSY gauge theories and integrable systems. We find complete agreement between the two approaches. In particular we show how the DV proposal allows the extraction of the exact eigenvalues of the adjoint scalar in the confining vacuum and hence computes all related condensates of the finite- $N$ gauge theory. We extend these results to include Leigh-Strassler deformations of the $\mathcal{N}=4$ theory.
\end{abstract}




\section{Introduction}

Theories with $\mathcal{N}=1$ supersymmetry in four dimensions are of considerable interest. Apart from their potential phenomenological relevance, they provide a field theoretic laboratory where physical phenomena such as confinement and dynamical symmetry breaking can be studied in a controlled setting. This is possible largely because these theories contain a special class of observables whose dependence on the parameters of the theory is constrained to be holomorphic. These quantities, which include the vacuum expectation values of chiral operators and the tensions of BPS saturated domain walls, are governed by a holomorphic superpotential which can sometimes be determined exactly. Although exact results for many different models have been obtained, a general recipe for computing the superpotential for an arbitrary $\mathcal{N}=1$ model has remained elusive. In recent work [1-4], Dijkgraaf and Vafa (DV) have proposed a solution to this problem which applies to a large class of $\mathcal{N}=1$ theories. Specifically, they claim that the superpotentials for these theories can be extracted from the planar limit of a certain holomorphic matrix integral. In this paper, we will perform a detailed test of their proposal against known results for a family of $\mathcal{N}=1$ preserving deformations of $\mathcal{N}=4$ SUSY Yang-Mills theory with gauge group $S U(N)$ (or $U(N)$ ). For this family of theories there is an alternative method for computing the exact superpotential, given in [5-8]. In fact, the superpotential can be deduced from the value of the Lax matrix of a certain classical integrable system in its equilibrium position. Remarkably, we will find that these two seemingly very different recipes give exactly the same result. The comparison will also shed light on both approaches. In the remainder of this introductory section, we will review both the DV proposal and the results of [8] based on the connection to integrable systems and present our main conclusions.

We begin by considering $\mathcal{N}=4$ SUSY Yang-Mills theory with gauge group $G=$ $S U(N)$ and bare complex coupling $\tau=4 \pi i / g_{Y M}^{2}+\theta / 2 \pi$. In the standard language of $\mathcal{N}=1$ superfields, the theory contains three adjoint-valued chiral multiplets, which we will label $\Phi^{+}, \Phi^{-}$and $\Phi$. We will consider the deformation of the $\mathcal{N}=4$ theory obtained by adding an $\mathcal{N}=2$ preserving mass term for $\Phi^{ \pm}$, together with a general polynomial superpotential for $\Phi$ which preserves $\mathcal{N}=1$ supersymmetry.The classical superpotential then reads,

$$
W(\Phi)=\operatorname{Tr}\left(i \Phi\left[\Phi^{+}, \Phi^{-}\right]+\Phi^{+} \Phi^{-}+\sum_{p=2}^{N} g_{p} \Phi^{p}\right)
$$

Setting $g_{p}=0$ for $p \geq 3$ corresponds to the $\mathcal{N}=1^{*}$ deformation but we will not limit ourselves to this case. 
This theory already has a rich vacuum structure at the classical level. In particular, there is a large set of classical vacua in which the adjoint scalar fields acquire vacuum expectation values (VEVs) spontaneously breaking some or all of the gauge symmetry. In this paper we will focus on SUSY vacua in which the full $S U(N)$ gauge symmetry is unbroken. At the classical level this corresponds to setting the adjoint scalar VEVs to zero ${ }^{1}$. The qualitative behaviour of the corresponding quantum theory in these vacua is well known: at energy scales much less than the mass scale set by the deformations, the chiral multiplets decouple leaving the theory of a single $S U(N)$ vector multiplet which is in the same universality class as $\mathcal{N}=1$ SUSY Yang-Mills. The theory is therefore in a confining phase with a mass gap and gluino condensation. There are $N$ supersymmetric vacua denoted $|k\rangle$ with $k=0,1,2, \ldots, N-1$, each with a non-zero expectation value for the gluino bilinear which is the lowest component of the superfield $S=\operatorname{Tr}\left(W_{\alpha} W^{\alpha}\right) / 32 \pi^{2}$. In fact, the action of S-duality, inherited from the underlying $\mathcal{N}=4$ theory, dictates that the vacuum states $|k\rangle$ and $|k+1\rangle$ are simply related by a $2 \pi$ shift in the vacuum angle: $\tau \rightarrow \tau+1$. Hence it will suffice to consider the $k=0$ vacuum, $|0\rangle$, in the following. Our aim will be to determine the exact effective superpotential in this vacuum, $\left\langle 0\left|W_{\text {eff }}\right| 0\right\rangle$. The vacuum expectation values of the operators $u_{p}=\operatorname{Tr} \Phi^{p}$ can then be obtained by differentiating with respect to the parameters $g_{p}$ appearing in (1.1).

\subsection{The integrable system approach}

An exact formula for the effective superpotential can be obtained from a slight extension of the results presented in [8]. This approach relies on the connection between SUSY gauge theories and integrable systems first discovered in [9-11]. In the present case the relevant integrable system is the elliptic Calogero-Moser (ECM) system. This is a system of $N$ non-relativistic particles with complex positions and momenta, $X_{j}$ and $P_{j}, j=1,2, \ldots, N$, which live on a torus $E_{\tau}$ of complex structure $\tau$ and its tangent space respectively. Time evolution is governed by the quadratic Hamiltonian,

$$
H_{2}=\sum_{j=1}^{N} \frac{P_{j}^{2}}{2}+\sum_{j>k} \wp\left(X_{j}-X_{k}\right)
$$

where $\wp$ is the Weierstrass function (see Appendix A.1) which is elliptic with respect to the torus $E_{\tau}$. The system has a Lax formulation involving two $N \times N$ matrices $L_{j k}$ and $M_{j k}$, in terms of which Hamilton's equations can be rewritten as $i \dot{L}=[M, L]$. This immediately implies the existence of $N$ conserved quantities $H_{p}=\operatorname{Tr} L^{p}$ for

\footnotetext{
${ }^{1}$ In general, the adjoint scalar fields can and will acquire VEVs in the quantum theory without breaking the gauge symmetry.
} 
$p=1, \ldots, N$. These quantities can also be thought of as a set of Poisson-commuting Hamiltonians each generating a distinct time-evolution of the system.

We will now state the relation between the deformed $\mathcal{N}=4$ theory and the ECM theory (for more details see [8]). Firstly, for each value of $p$, the conserved quantity $H_{p}$ is identified with the expectation value of the operator $u_{p}=\operatorname{Tr} \Phi^{p}$ introduced above ${ }^{2}$. As explained in [6], the supersymmetric vacua of the theory then correspond to the equilibrium configurations of the ECM particles. In particular, for the theory with an arbitrary polynomial deformation, as in Eq.(1.1) we must consider equilibrium configurations with respect to the time-evolution generated by the Hamiltonian $H=\sum_{p=2}^{N} g_{p} H_{p}$.

In the general case, finding these configurations is not straightforward. However, the massive vacua of the theory have a very special property: they correspond to static configurations which simultaneously stationarize each of the Hamiltonians ${ }^{3}$ $H_{p}$, for $p=2, \ldots, N$. In particular, the vacuum $|0\rangle$ introduced above corresponds to a configuration where the particles are evenly spaced around one cycle of the torus: $X_{j}=(2 \pi i \tau / N) j-i \pi \tau(N-1) / N ; P_{j}=0$ for $j=1, \ldots, N$. As each Hamiltonian is stationary, the same configuration corresponds to a SUSY vacuum for all values of the deformation parameters. The eigenvalues of the Lax matrix $L_{a b}$ in this equilibrium configuration were calculated explicitly in [8]. The result is

$$
\tilde{\lambda}_{j}=-\frac{1}{2} \frac{\theta_{3}^{\prime}(\pi / 2-\pi j / N \mid \tau / N)}{\theta_{3}(\pi / 2-\pi j / N \mid \tau / N)}
$$

where $j=1,2, \ldots, N$. The vacuum expectation values of the operators, $u_{p}$, can then be determined via the relation $\left\langle 0\left|u_{p}\right| 0\right\rangle=\sum_{j=1}^{N} \tilde{\lambda}_{j}^{p}$. As the resulting formulae are completely independent of the deformation parameters $g_{p}$, we find that that the vacuum value of the effective superpotential is simply given by,

$$
\left\langle 0\left|W_{\text {eff }}\right| 0\right\rangle=\sum_{p=2}^{N} g_{p}\left\langle 0\left|u_{p}\right| 0\right\rangle=\sum_{p=2}^{N} \sum_{j=1}^{N} g_{p} \tilde{\lambda}_{j}^{p}
$$

\footnotetext{
${ }^{2}$ To restrict to the $S U(N)$ theory where $u_{1}$ vanishes we consider the ECM system in its centerof-momentum frame where $H_{1}=\sum_{j=1}^{N} P_{j}=0$.

${ }^{3}$ In the field theory this is related to the fact that massive vacua preserving $\mathcal{N}=1$ supersymmetry correspond to the maximal degenerations of the Riemann surface which governs the Coulomb branch of the $\mathcal{N}=2$ theory obtained by setting all the deformation parameters to zero. However, it can also be demonstrated directly using the Lax representation for the equations of motion of the ECM system. This is shown in Appendix B.
} 


\subsection{The Dijkgraaf-Vafa Approach}

Dijkgraaf and Vafa have proposed an exact recipe for calculating the exact effective superpotential $W_{\text {eff }}$ as a function of the gluino condensate (the expectation value of the superfield $S$ ). Specifically, they relate the superpotential to the following holomorphic matrix integral:

$$
\mathcal{Z}=\int[d \Phi]\left[d \Phi^{+}\right]\left[d \Phi^{-}\right] \exp \left(-\frac{1}{g_{s}} W(\Phi)\right)
$$

where $g_{s}$ is a coupling constant. The integral is to be understood as a multi-variable contour integral. As for any adjoint $S U(N)$ theory the free energy $\mathcal{F}$ has a 't Hooft expansion where the Feynman diagrams are organised according to their genus $g \geq 0$.

$$
\mathcal{F}=\sum_{g \geq 0} g_{s}^{2 g-2} \mathcal{F}_{g}(\lambda)
$$

where $\lambda=g_{s} N$ is the matrix model 't Hooft coupling. DV predict that the exact superpotential of the above $\mathcal{N}=1$ perturbation of the $\mathcal{N}=4$ theory only depends on the leading term in this expansion: $\mathcal{F}_{0}(\lambda)$. This term may be evaluated by taking the 't Hooft limit of the matrix model, $N \rightarrow \infty, g_{s} \rightarrow 0$ with $\lambda$ held fixed, which isolates the planar Feynman diagrams. Fortunately the matrix model can be solved exactly in this limit using standard saddle-point technology. In the case of a quadratic deformation, the solution has been already been given by Kazakov, Kostov and Nekrasov [13]. Generalization of their solution to an arbitrary polynomial deformation is straightforward and is given below.

The proposed dictionary between matrix model and field theory quantities identifies the 't Hooft coupling $\lambda=g_{s} N$ with the expectation value of the gluino bilinear superfield $S$. From now on we will use the symbol $S$ to denote both the matrix model 't Hooft coupling and the gluino condensate of the field theory and assume that the interpretation is clear from the context. The resulting expression for the field theory superpotential is,

$$
W_{\text {eff }}(S)=N \frac{\partial \mathcal{F}_{0}}{\partial S}-2 \pi i \tau S
$$

The supersymmetric vacua may be found be extremising $W_{\text {eff }}$ as a function of S. The expectation value of $W_{\text {eff }}$ in each vacuum is simply its value at the corresponding the extremum. The main aim of this paper is to compare the resulting formulae with the exact field theory predictions Eqs. (1.3) and (1.4). We find that the DV prescrip-

tion allows us to extract the field theory eigenvalues $\tilde{\lambda}_{j}$ and we find precise agreement between the two approaches, for arbitrary values of the deformation parameters $g_{p}$, 
up to the known ambiguities in the definition of the operators $u_{p}$ [5]. Agreement in the case of a quadratic superpotential has previously been checked in [4].

Several features of our result are worthy of further comment. A remarkable consequence of Dijkgraaf and Vafa's proposal is that the holomorphic quantities of the deformed $\mathcal{N}=4$ theory are large- $N$ exact. Although this property is far from obvious in the integrable systems approach it nevertheless seems to be true that $1 / N$ corrections to all physical quantities vanish up to the usual operator mixing ambiguities! On another hand there are some features of the field theory results which are not apparent at the outset in the DV prescription, for example the linear dependence of the exact superpotential (1.4) on the deformation parameters $g_{p}$. Nevertheless, we are able to prove that for the class of deformations considered the DV prescription automatically implies this linearity in the confining vacuum.

We also extend our study to include a different class of deformations of the $\mathcal{N}=4$ theory, namely the Leigh-Strassler deformations. We use the DV proposal to compute the exact effective superpotential for these deformations and extract the

eigenvalues $\tilde{\lambda}_{j}$ in the confining vacuum of the theory. Remarkably, we find once again that these are independent of the deformation parameters $g_{p}$.

\section{Superpotentials and eigenvalue distributions from matrix models}

According to the Dijkgraaf-Vafa proposal [1-3] superpotentials for a large class of $\mathcal{N}=1$ supersymmetric field theories at finite $N$ are computed by corresponding large- $N$ matrix models. Assuming the validity of this conjecture for arbitrary deformations of the $\mathcal{N}=4$ theory, we will demonstrate that for such deformations, in the confining vacuum of the resulting theory the matrix model in fact yields the correct eigenvalue distribution for the adjoint scalar field in the theory. This tests the Dijkgraaf-Vafa proposal for a large class of deformations of the $\mathcal{N}=4$ theory. Furthermore, it provides a general prescription for extracting QFT eigenvalue distributions from the matrix model and also demonstrates that the matrix model computes all condensates in the holomorphic sector of the theory.

\subsection{Deformations of $\mathcal{N}=4$ theory}

In the $\mathcal{N}=1$ language, the $\mathcal{N}=4 U(N)$ theory with coupling constant $\tau \equiv$ $4 \pi i / g_{Y M}^{2}+\theta / 2 \pi$ has three adjoint chiral superfields $\Phi^{+}, \Phi^{-}$and $\Phi$. We consider a 
general class of deformations of the $\mathcal{N}=4$ theory specified by a tree level superpotential

$$
W=\frac{1}{g_{Y M}^{2}} \operatorname{Tr}\left[i \Phi\left[\Phi^{+}, \Phi^{-}\right]+\Phi^{+} \Phi^{-}+V(\Phi)\right]
$$

where $V(\Phi)$ is a general polynomial

$$
V(\Phi) \equiv \sum_{p} g_{p} \Phi^{p}
$$

Note that for the sake of simplicity we have set any and all mass scales to unity. (For $V(\Phi)=\Phi^{2}$ we obtain the $\mathcal{N}=1^{*}$ theory.)

As a direct consequence of the proposal of Dijkgraaf and Vafa [1-3], in a given vacuum the exact effective superpotential for the above class of deformations of the $\mathcal{N}=4$ theory is computed by the planar diagram expansion, i.e. a large- $N$ limit, of the three-matrix model partition function expanded around that vacuum

$$
Z=\int\left[d \Phi^{+}\right]\left[d \Phi^{-}\right][d \Phi] \exp -\frac{1}{g_{s}} \operatorname{Tr}\left[i \Phi\left[\Phi^{+}, \Phi^{-}\right]+\Phi^{+} \Phi^{-}+V(\Phi)\right]
$$

with $\Phi^{+}=\left(\Phi^{-}\right)^{\dagger}$.

The confining vacua of the field theory correspond to the classical solution $\Phi=$ $\Phi^{ \pm}=0$ which preserves the full $U(N)$ gauge symmetry. In order to implement the Dijkgraaf-Vafa proposal for extracting the field theory superpotential in the confining vacua we therefore need to solve the matrix model Eq. (2.3) around the trivial vacuum $\Phi=\Phi^{ \pm}=0$. As noted for example in [13], the key fact that permits the solution of these matrix models is that one may integrate out $\Phi^{ \pm}$exactly to obtain a one-matrix integral to be solved in the large- $N$ limit

$$
Z=\int[d \Phi] \frac{e^{-\frac{1}{g_{s}} \operatorname{Tr} V(\Phi)}}{\operatorname{det}\left(\operatorname{Adj}_{\Phi}+i\right)} .
$$

\subsection{Solution of the large- $N$ matrix model}

The above one-matrix model actually becomes tractable in the large- $N$ limit by going to an eigenvalue basis and performing a large- $N$ saddle-point approximation to the integral. The details of this procedure have been extensively discussed in the literature. We refer the reader to [13], and references therein, for details.

The main feature of the solution is that the eigenvalues $\lambda_{j}$ of $\Phi$ interact via a repulsive effective potential and form a continuum in the large- $N$ limit and condense into a cut along the real axis. The actual extent of the cut and the density of 
eigenvalues $\rho(\lambda)$ along the cut is self-consistently determined by the saddle point equation in terms of the parameters of the deformation $V(\Phi)$ and the matrix model 't Hooft coupling $S=g_{s} N$. The saddle point equation in the large- $N$ limit is most conveniently written in terms of the resolvent function

$$
\omega(z)=\int_{-\alpha}^{+\alpha} \frac{\rho(\lambda)}{z-\lambda} d \lambda \quad \lambda \in[-\alpha, \alpha] ; \quad \int_{-\alpha}^{+\alpha} \rho(\lambda) d \lambda=1 .
$$

The resolvent $\omega(z)$ is an analytic function on the complex $z$-plane whose only singularity is a branch cut with the discontinuity across the cut given precisely by the spectral density

$$
\omega(\lambda+i \epsilon)-\omega(\lambda-i \epsilon)=-2 \pi i \rho(\lambda) ; \quad \lambda \in[-\alpha, \alpha]
$$

The saddle point equation essentially encodes the condition that the force on a test eigenvalue due to the remaining large- $N$ equilibrium distribution of eigenvalues vanishes along the cut where the eigenvalues condense. The matrix model effective action yields this force on a test eigenvalue at position $z$ in the complex plane as

$$
F(z)=\frac{1}{2 i}\left[G\left(z+\frac{i}{2}\right)-G\left(z-\frac{i}{2}\right)\right]
$$

where

$$
G(z)=U(z)+i S\left(\omega\left(z+\frac{i}{2}\right)-\omega\left(z-\frac{i}{2}\right)\right) .
$$

and $U(z)$ is a polynomial in $z$ such that

$$
V^{\prime}(z)=\frac{1}{i}\left[U\left(z+\frac{i}{2}\right)-U\left(z-\frac{i}{2}\right)\right] .
$$

The analytic properties of the function $G(z)$ are central to the solution of the model and its subsequent physical interpretation. The defining equation for $G(z)$ Eq. (2.8) implies that $G(z)$ is an analytic function with two branch cuts between $\left[-\alpha+\frac{i}{2}, \alpha+\frac{i}{2}\right]$ and $\left[-\alpha-\frac{i}{2}, \alpha-\frac{i}{2}\right]$. The matrix model saddle point equation identifies the values of $G(z)$ along the two cuts via

$$
G\left(\lambda+\frac{i}{2} \pm i \epsilon\right)=G\left(\lambda-\frac{i}{2} \mp i \epsilon\right) ; \quad \lambda \in[-\alpha, \alpha] .
$$

Thus the saddle point equation may be viewed as a 'gluing' condition for the two cuts on the $z$-plane. In addition $G(z)=G\left(z^{*}\right)^{*}$ and $G(z)=G(-z)$. The function $G(z)$ is then uniquely determined by its asymptotic behaviour at $z \rightarrow \infty$ which is specified by the polynomial $U(z)$ in Eq. (2.8).

Given the density of eigenvalues $\rho(\lambda)$ in the matrix model one may use it to compute expectation values of various quantities in the matrix model. However, at the 

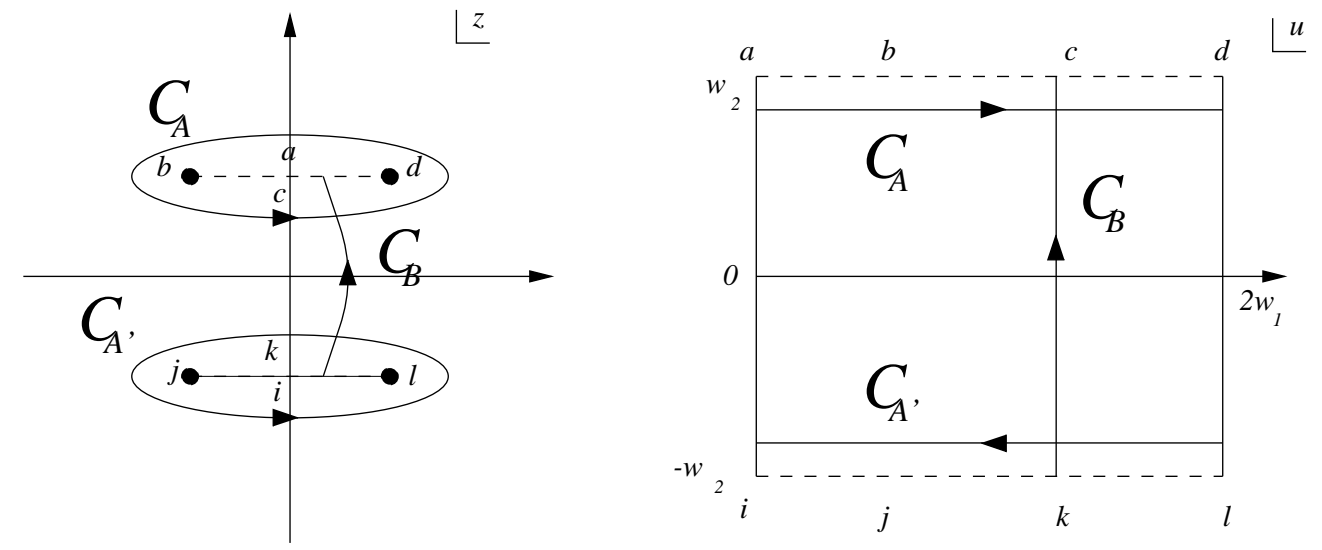

Figure 1: The $z$-plane and its pre-image in the $u$-plane. The cuts in the $z$-plane are indicated by the dotted lines with Roman letters to dictate how they are mapped from the $u$-plane. Three representative closed contours $\mathcal{C}_{A}, \mathcal{C}_{B}$ and $\mathcal{C}_{A^{\prime}}$ are illustrated.

outset we would like to point out these expectation values are distinct from vacuum expectation values of the corresponding observables in field theory. In particular, expectation values of the quantities $\operatorname{Tr} \Phi^{n}$ in the matrix model are defined in the obvious way:

$$
\left\langle\left\langle\operatorname{Tr} \Phi^{n}\right\rangle\right\rangle=\int_{-\alpha}^{\alpha} \rho(\lambda) \lambda^{n} d \lambda .
$$

Using the formulae above one can relate these expectation values to the terms in the large- $z$ expansion of $G(z)$. However these expectation values $\left\langle\left\langle\operatorname{Tr} \Phi^{n}\right\rangle\right\rangle$ are not to be identified with the corresponding condensates $\left\langle\operatorname{Tr} \Phi^{n}\right\rangle$ in the field theory,

$$
\left\langle\operatorname{Tr} \Phi^{n}\right\rangle_{Q F T} \neq\left\langle\left\langle\operatorname{Tr} \Phi^{n}\right\rangle\right\rangle
$$

\subsection{Emergence of the $\tilde{\tau}$-torus}

The two-cut structure of the $z$-plane (from the analytic properties of $G(z)$ ) and the gluing conditions Eq. (2.10) uniquely specify a torus which we denote by $E_{\tilde{\tau}}$ where $\tilde{\tau}$ is defined to be the complex structure parameter of this torus. The contour $\mathcal{C}_{A}$ in the $z$-plane enclosing the cut $\left[-\alpha+\frac{i}{2}, \alpha+\frac{i}{2}\right]$ maps to the $A$-cycle of the torus while the contour $\mathcal{C}_{B}$ joining the two cuts maps to the $B$-cycle of the torus. We also define $\mathcal{C}_{A^{\prime}}$ as the contour enclosing the lower cut. Note that $\mathcal{C}_{A}+\mathcal{C}_{A^{\prime}}$ can be pulled away from the cuts to enclose the point at infinity. These contours in the $z$-plane are indicated in Figure 1.

The torus $E_{\tilde{\tau}}$ may be thought of as usual, as a quotient of the complex plane, say the $u$-plane (this auxiliary variable $u$ will lead to the required elliptic parametriza- 
tion), by a lattice with periods $2 \omega_{1}$ and $2 \omega_{2}$ with $\omega_{2} / \omega_{1}=\tilde{\tau}$. The physical significance of this torus will become apparent upon implementing the procedure of [1-3] for computing the field theory superpotential. In fact $\tilde{\tau}$ will be identified with $\tau / N$, signalling a low-energy $S L(2, \mathbb{Z})$ duality symmetry in $\tau / N$ where $\tau$ is the coupling of the $\mathcal{N}=4$ theory. This duality, dubbed $\tilde{S}$-duality in [5] is distinct from the $S L(2, \mathbb{Z})$ symmetry of the $\mathcal{N}=4$ theory although it is of course a consequence of the latter.

The map $z(u)$ from the $u$-plane to the $z$-plane is specified by the requirements that going around $\mathcal{C}_{A}$ (the $A$-cycle of the torus) returns $z$ to its original value, while traversing the contour $\mathcal{C}_{B}$ (the $B$-cycle of the torus) causes $z$ to jump by an amount $i$, which is the distance between the two cuts in the $z$-plane. Both these operations leave $G$ unchanged which is therefore an elliptic function on the $u$-plane. Thus

$$
\begin{array}{ll}
A-\text { cycle }: & z\left(u+2 \omega_{1}\right)=z(u) ; \quad G\left(z\left(u+2 \omega_{1}\right)\right)=G(z(u)), \\
B-\text { cycle }: & z\left(u+2 \omega_{2}\right)=z(u)+i ; \quad G\left(z\left(u+2 \omega_{2}\right)\right)=G(z(u)) .
\end{array}
$$

These requirements (along with the power law behaviour of $G(z)$ at large $z$ ) specify $z(u)$ uniquely and we find that

$$
z(u)=-\frac{\omega_{1}}{\pi}\left[\zeta(u)-\frac{\zeta\left(\omega_{1}\right)}{\omega_{1}} u\right] \equiv-\frac{1}{2} \frac{\theta_{1}^{\prime}\left(\frac{\pi u}{2 \omega_{1}} \mid \tilde{\tau}\right)}{\theta_{1}\left(\frac{\pi u}{2 \omega_{1}} \mid \tilde{\tau}\right)} .
$$

Here $\zeta(z)$ is the Weierstrass zeta function and $\theta_{1}$ is the Jacobi theta function (see [14] and the Appendix for details). Note that the map from the torus to the eigenvalue plane is completely independent of the choice of the field theory deformation $V(\Phi)$. Different deformations imply different asymptotic properties of the elliptic function $G(z(u))$ as $z \rightarrow \infty$ and hence lead to elliptic functions with different orders.

\subsubsection{An Example}

For the simplest deformation, i.e. the quadratic one corresponding to the $N=1^{*}$ theory, the asymptotics determine $G(z(u))$ uniquely:

$$
V(\Phi)=\Phi^{2}: \quad G(z(u))=\frac{\omega_{1}^{2}}{\pi^{2}}\left[\wp(u)-2 \frac{\zeta\left(\omega_{1}\right)}{\omega_{1}}\right]
$$

$G(z(u))$ will involve higher powers of the Weierstrass- $\wp$ function for more complicated deformations.

Let us see how in the simplest example which was first discussed and solved in $[3,4], V(\Phi)=\Phi^{2}, \tilde{\tau}$ gets identified with $\tau / N$. To see this we integrate the oneform $G(z) d z$ over the $A$-cycle and $B$-cycle to obtain the total number of eigenvalues $S$ 
and $\partial \mathcal{F}_{0} / \partial S$ respectively, the latter being the variation in the genus zero free energy of the matrix model upon transporting an eigenvalue to infinity,

$$
\begin{aligned}
& \Pi_{A}=2 \pi i S=-i \int_{A} G(z(u)) \frac{d z(u)}{d u} d u=\frac{1}{12} \frac{d E_{2}(\tilde{\tau})}{d \tilde{\tau}} \\
& \Pi_{B}=\frac{\partial \mathcal{F}_{0}}{\partial S}=-i \int_{B} G(z(u)) \frac{d z(u)}{d u} d u=\frac{\tilde{\tau}}{12} \frac{d E_{2}(\tilde{\tau})}{d \tilde{\tau}}-\frac{1}{12} E_{2}(\tilde{\tau}) .
\end{aligned}
$$

Then the effective superpotential $W_{\text {eff }}$ for the $\mathcal{N}=1^{*}$ theory in the confining vacuum is obtained by extremizing the following expression with respect to $S$,

$$
W_{\mathrm{eff}}=N \Pi_{B}-\tau \Pi_{A}
$$

Using our expressions for the respective integrals over the cycles it is easy to see that,

$$
\frac{\partial W_{\mathrm{eff}}}{\partial S}=0 \Longrightarrow \tilde{\tau}=\frac{\tau}{N}
$$

and

$$
W_{\text {eff }}=-\frac{N}{12} E_{2}(\tau / N)
$$

Upon restoring the mass scales in the problem this result agrees precisely, up to an additive constant with the expressions of $[6,7]$. The fact that $S$ in Eq.(2.16a) automatically comes out as the $\tau$-derivative of the effective superpotential is in line with the identification of $S$ with gluino condensate of the gauge theory.

The identification of the complex structure $\tilde{\tau}$ of the matrix model torus with $\tau / N$ in the confining vacuum of the field theory in fact holds for all possible choices of $V(\Phi)$ and indeed, seems to work for a completely different class of deformations namely the Leigh-Strassler deformations which will be discussed in detail below. The emergence of a torus with complex structure $\tau / N$, controlling the low-energy physics of the confining vacua of deformations of the $\mathcal{N}=4$ theory, has been well understood from the point of view of Seiberg-Witten theory in $[5,12]$. It is quite remarkable to see this structure emerge from the matrix model.

\subsection{QFT eigenvalues and general superpotentials}

Before embarking on a discussion of the eigenvalues below, we should emphasize a subtle point that will emerge in due course. It will turn out that the distribution of eigenvalues in the matrix model described by the density $\rho(\lambda)$ is not to be identified with the distribution of eigenvalues of $\Phi$ in the field theory at large $N$. In fact, the main result of this section is that the Dijkgraaf-Vafa proposal is tantamount to iden-

tifying the eigenvalues of the adjoint scalar in the confining vacuum of the field theory 
with the function $z(u)$ in Eq. (2.14) (after a simple shift of its argument), distributed evenly over a cycle on the $E_{\tilde{\tau}}$ torus. In contrast the matrix model eigenvalues are not uniformly distributed along this cycle.

The function $z(u)$ that we have obtained above is a map from the torus $E_{\tilde{\tau}}$ to the plane of eigenvalues $z$. In particular, $z(u)$ maps the $A$ cycle of the torus to the cut enclosed by $\mathcal{C}_{A}$,

$$
\mathcal{C}_{A} \simeq z\left(2 \omega_{1} x+\omega_{2}\right)=-\frac{1}{2} \frac{\theta_{3}^{\prime}(\pi x \mid \tilde{\tau})}{\theta_{3}(\pi x \mid \tilde{\tau})}+\frac{i}{2} ; \quad x \in\left[-\frac{1}{2}, \frac{1}{2}\right] .
$$

This cut extends from $-\alpha+\frac{i}{2}$ to $\alpha+\frac{i}{2}$ and represents the possible values of the eigenvalues $\lambda$ of $\Phi$ shifted by $i / 2$ (the $i / 2$ shift is deduced simply from Eq. (2.8)). In view of this we define the function

$$
\lambda(x)=-\frac{1}{2} \frac{\theta_{3}^{\prime}(\pi x \mid \tilde{\tau})}{\theta_{3}(\pi x \mid \tilde{\tau})} ; \quad x \in\left[-\frac{1}{2}, \frac{1}{2}\right] .
$$

At first sight, this is simply a map from the $A$ cycle of the torus to the interval $[-\alpha, \alpha]$ in which the eigenvalues are distributed. However, we will soon find that $\lambda(x)$ has greater significance. Before we uncover this, we note that given the map $\lambda(x)$ we can ask how the eigenvalues of the matrix model are distributed along the $A$ cycle of the torus. The answer is rather complicated being given by

$$
\frac{d n}{d x}=\rho(\lambda(x)) \frac{d \lambda}{d x},
$$

with $-x_{0} \leq x \leq x_{0}$ where $\lambda\left(x_{0}\right)=\alpha{ }^{4}$

In contrast, we will now show how the proposal of [4] automatically implies that eigenvalues of $\Phi$ in the confining vacuum of the field theory at large $N$ are uniformly distributed over the $x$-interval $\left[-\frac{1}{2}, \frac{1}{2}\right]$ and are given precisely by the function $\lambda(x)$. To see this we simply note that the function $z^{\prime \prime}(u)=\frac{\omega_{1}}{\pi} \wp^{\prime}(u)$ is elliptic on the torus and thus the following integral vanishes

$$
\int_{\mathcal{C}_{A}+\mathcal{C}_{A}^{\prime}} G(z(u)) z^{\prime \prime}(u) d u=0 .
$$

Using the definition of $G(z)$ in Eq. (2.8) this implies

$$
S \int_{\mathcal{C}_{A}+\mathcal{C}_{A}^{\prime}}\left(\omega\left(z+\frac{i}{2}\right)-\omega\left(z-\frac{i}{2}\right)\right) z^{\prime \prime}(u) d u=i \int_{\mathcal{C}_{A}+\mathcal{C}_{A}^{\prime}} U(z) z^{\prime \prime}(u) d u .
$$

The periodicity of $z(u)$ along the $A$-cycle allows us to reduce the left hand side of this expression to a contour integral around $u=0$ picking out the residue at $u=0$.

\footnotetext{
${ }^{4}$ Notice that the $A$ cycle covers the interval $[-\alpha, \alpha]$ twice, so one must choose one of the branches.
} 
Using $z\left(2 \omega_{1} x \pm \omega_{2}\right)=\lambda(x) \pm \frac{i}{2}$, and Eq. (2.9) and the heat equation for the Jacobi theta functions (A.9) we find that

$$
S=-\frac{1}{2 i \pi} \frac{d}{d \tilde{\tau}} \int_{-1 / 2}^{1 / 2} V(\lambda(x)) d x
$$

with $\lambda(x)$ given by Eq. (2.21) Since the proposal of [4] implies the identification $\tilde{\tau}=\tau / N$ and $2 \pi i S=-d W_{\text {eff }} / d \tau$ so that $S$ is the gluino condensate, we must have

$$
W_{\mathrm{eff}}=N \int_{-1 / 2}^{1 / 2} V(\lambda(x)) d x ; \quad \lambda(x)=-\frac{1}{2} \frac{\theta_{3}^{\prime}(\pi x \mid \tilde{\tau})}{\theta_{3}(\pi x \mid \tilde{\tau})}
$$

This proves that the eigenvalues of the adjoint scalar $\Phi$ in the confining vacuum of the field theory at large $N$ are given by the function $\lambda(x)$ and are uniformly distributed over the interval $x \in\left[-\frac{1}{2}, \frac{1}{2}\right]$ independent of the deformation $V(\Phi)$. Note that, on the contrary the distribution of eigenvalues of the matrix model depends on the deformation in a complicated way.

We should also emphasize that the result (2.26) is also, according to Dijkgraaf and Vafa, true at finite $N$. At finite $N$ the eigenvalues of $\Phi$ in the confining vacuum have been computed from a completely different approach using the connection between $\mathcal{N}=1^{*}$ theories and integrable systems in [8]. The eigenvalues found in [8] depend on a discrete index $j$ and were given as

$$
\tilde{\lambda}_{j}=-\frac{1}{2} \frac{\theta_{3}^{\prime}(\pi / 2-\pi j / N \mid \tau / N)}{\theta_{3}(\pi / 2-\pi j / N \mid \tau / N)} ; \quad j=1, \ldots, N
$$

(Notice that these, as explained above, are not to be identified with the eigenvalues $\lambda_{j}$ of the matrix model!) The Dijkgraaf-Vafa prediction from the matrix model above in Eq. (2.26) agrees precisely with the results of [8] upon taking the large- $N$ limit where the discrete label $j$ in Eq.(2.27) is replaced by the continuous variable $x$. In particular the eigenvalues are uniformly distributed in $x$. This demonstrates that using the recipe of [1-3] the matrix model indeed computes the superpotentials for the entire class of deformations considered above, provided $N$ is large. Alternatively, for any given deformation it also computes all the condensates $\left\langle\operatorname{Tr} \Phi^{n}\right\rangle$ via

$$
\left\langle\operatorname{Tr} \Phi^{n}\right\rangle=N \int_{-1 / 2}^{1 / 2} \lambda^{n}(x) d x
$$

In concert with all our previous warning $\left\langle\operatorname{Tr} \Phi^{n}\right\rangle$, the condensate of the field theory is not to be identified with the expectation values in the matrix model (2.11):

$$
\left\langle\left\langle\operatorname{Tr} \Phi^{n}\right\rangle\right\rangle \neq\left\langle\operatorname{Tr} \Phi^{n}\right\rangle
$$


As we have already mentioned, the eigenvalues and hence all the condensates in the confining vacuum are completely independent of the actual choice of deformations. This is in complete accord with expectations from field theory. The deformations of the $\mathcal{N}=4$ theory that we have discussed may be thought of as $\mathcal{N}=1$ perturbations of the $\mathcal{N}=2^{*}$ theory. The confining vacua of the resulting theories arise from points on the Coulomb branch where the Seiberg-Witten curve for the $\mathcal{N}=2^{*}$ theory degenerates maximally into a torus of complex structure $\tau / N$. The location of such a point in moduli space given by condensates $\left\langle\operatorname{Tr} \Phi^{n}\right\rangle$, is completely independent of the choice of $V(\Phi)$. This is in fact expected to be true in all massive vacua of the $\mathcal{N}=1$ theory.

Although we have seen a remarkable agreement between the matrix model recipe and the field theory results of [8], strictly this agreement appears when the eigenvalues form a continuous distribution and can happen only in the large- $N$ limit of the field theory. Indeed, the appearance of integrals over a continuous label rather discrete sums in Eq. (2.26) suggests that we are really doing a large- $N$ calculation from the point of view of the field theory. So does the large- $N$ matrix model, or the planar graphs of the matrix model really compute the holomorphic sector of the finite- $N$ field theory? The resolution of this puzzle will be the subject of Section 3.

\subsection{Some explicit examples}

We have computed the superpotentials from the matrix model by explicitly obtaining $G(z(u))$ for $V(\Phi)=\Phi^{2}$ and $\Phi^{4}$ and computing $S, \partial \mathcal{F}_{0} / \partial S$ as the integrals of $G(z) d z$ over the $A$ and $B$ cycles. These results match with a direct computation of the superpotential using the eigenvalue distribution following Eq. (2.26). We quote the results for these cases below

(i) $V(\Phi)=\Phi^{2}$ :

$$
\begin{aligned}
G(z(u)) & =\frac{\omega_{1}^{2}}{\pi^{2}} \wp(u)-\frac{2 w_{1} \zeta\left(\omega_{1}\right)}{\pi^{2}} \\
\Pi_{A} & =\frac{d h(\tilde{\tau})}{d \tilde{\tau}} ; \quad \Pi_{B}=\tilde{\tau} \frac{d h(\tilde{\tau})}{d \tilde{\tau}}-h(\tilde{\tau}) . \\
W_{\mathrm{eff}} & =-N h(\tau / N)=-\frac{N}{12} E_{2}(\tau / N) .
\end{aligned}
$$

The additive normalization of $W_{\text {eff }}$ can be fixed by a direct computation of Eq. (2.26) which gives

$$
W_{\text {eff }}=N \int_{-1 / 2}^{1 / 2} \lambda^{2}(x) d x=-\frac{N}{12}\left(E_{2}(\tau / N)-1\right)
$$


(ii) $V(\Phi)=\Phi^{4}$ :

$$
\begin{aligned}
G(z(u))=\frac{\omega_{1}^{4}}{\pi^{4}} \wp(u)^{2}+ & \frac{\omega_{1}^{2}}{2 \pi^{4}}\left(\pi^{2}-8 \omega_{1} \zeta\left(\omega_{1}\right)\right) \wp(u) \\
& -\frac{\omega_{1}}{6 \pi^{4}}\left(g_{2} \omega_{1}^{3}+6 \pi^{2} \zeta\left(\omega_{1}\right)-36 \omega_{1} \zeta\left(\omega_{1}\right)^{2}\right), \\
\Pi_{A}= & \frac{d h(\tilde{\tau})}{d \tilde{\tau}} ; \quad \Pi_{B}=\tilde{\tau} \frac{d h(\tilde{\tau})}{d \tilde{\tau}}-h(\tilde{\tau}) . \\
W_{\text {eff }}= & -N h(\tau / N)=\frac{N}{720}\left(10 E_{2}(\tau / N)^{2}-E_{4}(\tau / N)-30 E_{2}(\tau / N)\right) .
\end{aligned}
$$

The additive normalization of $W_{\text {eff }}$ can be fixed by a direct computation of Eq. (2.26) which gives

$$
W_{\text {eff }}=N \int_{-1 / 2}^{1 / 2} \lambda^{4}(x) d x=\frac{N}{720}\left(10 E_{2}(\tau / N)^{2}-E_{4}(\tau / N)-30 E_{2}(\tau / N)+21\right) .
$$

\subsection{Summary}

In summary, in this section we have verified that the Dijkgraaf-Vafa prescription for computing $\mathcal{N}=1$ superpotentials from matrix models works for a large class of deformations of the $\mathcal{N}=4$ theory. In particular it appears to provide a recipe for extracting quantum field theory eigenvalue distributions from the matrix model which therefore permits an exact computation of all higher condensates and superpotentials for general deformations of the type discussed above. Our check of this proposal rests on the precise agreement between these field theory eigenvalues extracted from the matrix model, and those computed independently from a completely different approach in [8].

It must be emphasized that the field theory eigenvalue distributions are not identical to the matrix model distributions. The spectral densities of the two systems

are different although there exists a recipe for extracting the QFT distributions from those of the matrix model.

\section{Large $N$ versus finite $N$}

As we have mentioned, one remarkable consequence of the Dijkgraaf-Vafa hypothesis is that the exact superpotential is captured by a large- $N$ analysis. This presents a real puzzle: we have already remarked that the matrix model yields the distribution of eigenvalues of $\Phi$ which agrees with the large- $N$ limit of the distribution computed 
from the integrable system approach in [8]; however, surely when we calculate the condensates $\left\langle\operatorname{Tr} \Phi^{n}\right\rangle$ using the finite- $N$ distribution of [8], we will find differences from the large- $N$ result.

Put another way, the large- $N$ matrix model (DV) result for the condensates (2.28) are given by an integral along one of the cycles on the torus which can be written in terms of an integral over theta functions using Eqs. (2.21) and (2.28):

$$
\left\langle\operatorname{Tr} \Phi^{n}\right\rangle_{\mathrm{DV}}=N \int_{-1 / 2}^{1 / 2}\left(-\frac{1}{2} \frac{\theta_{3}^{\prime}(\pi x \mid \tau / N)}{\theta_{3}(\pi x \mid \tau / N)}\right)^{n} d x
$$

while, in the same normalization, the condensates at finite- $N$ can be calculated from the exact eigenvalues that follow from the integrable system. In the confining vacuum these are given in Eq. (2.27). Hence, the condensate which follows from the integrable system analysis is

$$
\left\langle\operatorname{Tr} \Phi^{n}\right\rangle_{\text {int }}=\sum_{j=1}^{N}\left(-\frac{1}{2} \frac{\theta_{3}^{\prime}(\pi / 2-\pi j / N \mid \tau / N)}{\theta_{3}(\pi / 2-\pi j / N \mid \tau / N)}\right)^{n} .
$$

Clearly, (3.1) and (3.2) differ at finite $N$ and so, one might think, that at finite $N$ the whole Dijkgraaf-Vafa hypothesis is called into question.

However, it is well-known that the condensates of the $\mathcal{N}=1^{*}$ theory are plagued by mixing ambiguities $[5,15]$. There is no canonical definition of the operator $\Phi^{n}$ and different approaches can lead to expressions which differ by admixtures of $\Phi^{p}$, $p<n$ (including the identity operator $p=0$ ). What is important, however, is that the mixing coefficients should be vacuum independent. This means that the mixing coefficients should only depend on modular functions involving the torus whose complex structure is the bare coupling $\tau$ rather than the effective coupling in the confining vacuum $\tau / N$. It simply would not make sense if the mixing coefficients between two approaches depended on the vacuum - in the present case the confining one. Having appreciated the possibility of mixing we can now see how the DijkgraafVafa hypothesis can be correct even though (3.1) and (3.2) are manifestly different at finite $N$. The resolution is that definitions of $\left\langle\operatorname{Tr} \Phi^{n}\right\rangle_{\mathrm{DV}}$ and $\left\langle\operatorname{Tr} \Phi^{n}\right\rangle_{\text {int }}$ differ by vacuum independent mixings.

Although we have no general proof of this, we can present very strong arguments for its verisimilitude. Firstly consider $n=2$. In this case, the sum (3.2) gives

$$
\left\langle\operatorname{Tr} \Phi^{2}\right\rangle_{\mathrm{int}}=-\frac{N}{12}\left(E_{2}(\tau / N)+(N-2) E_{2}(\tau)-(N-1) E_{2}(\tau / 2)\right) .
$$

Notice that (3.3) actually vanishes for $N=2$ which follow from the fact that the two eigenvalues vanish in this case. Notice also that it differs from (2.31) by terms 
which involve modular functions of $\tau$ and $\tau / 2$, rather than $\tau / N$ and, therefore, one suspects that they differ by a vacuum independent constant which corresponds to mixing with the identity operator.

At this point we should remind the reader that the class of theories we are considering have a large number of vacua with a mass gap, where the theory is realised in different phases. The canonical examples are the Higgs vacuum where the gauge group is broken completely by the Higgs mechanism, and the confining vacuum where classically the full gauge symmetry is unbroken although quantum mechanically there are no massless states due to strong coupling effects which cause the elementary degrees of freedom to confine and generate a mass gap. It is well-known that the Higgs and confining vacua are actually related by an $S$-duality, $\tau \rightarrow-1 / \tau$, while more general modular transformations map to other massive vacua $[6,7,17]$. This provides us with a natural definition for the condensates.

One way to disentangle the operator ambiguities is to note there exists a natural definition of the condensates, which we denote simply as $\left\langle\operatorname{Tr} \Phi^{n}\right\rangle$, that have "good" modular properties. What we mean by this is that the value of the condensate in the Higgs vacuum is given by taking the value in the confining vacuum with the $S$ duality replacement $\tau \rightarrow-1 / \tau$ and then by multiplying by the factor $\tau^{-n}[5,6]$. The values in other vacua are also related by other modular transformations. That such a basis of operators with good modular properties should exist follows ultimately from the fact that we are considering a deformation of the $\mathcal{N}=4$ theory, which is self-dual under modular transformations, by operators which can be chosen to have a definite weight. By assigning a compensating modular weight to the couplings the resulting deformed theory is then modular invariant. However, the vacua of the theory spontaneously break the modular symmetry. The modular symmetry then simply permute the vacua. We can also establish the existence of the modular covariant basis of operators directly from the integrable system: this tells us how $\left\langle\operatorname{Tr} \Phi^{n}\right\rangle$ is related to $\left\langle\operatorname{Tr} \Phi^{n}\right\rangle_{\text {int }}$ and therefore, using (3.3) and its generalization to arbitrary deformation, how $\left\langle\operatorname{Tr} \Phi^{n}\right\rangle$ is related to $\left\langle\operatorname{Tr} \Phi^{n}\right\rangle_{\mathrm{DV}}$.

For the $\Phi^{2}$ operator, the modular covariant definition of the condensate is related to the matrix one by the vacuum independent - so involving modular functions of $\tau$ rather than $\tau / N$ - shift

$$
\left\langle\operatorname{Tr} \Phi^{2}\right\rangle=\left\langle\operatorname{Tr} \Phi^{2}\right\rangle_{\mathrm{DV}}+\frac{N}{12}\left(N E_{2}(\tau)-1\right) .
$$

This then yields the modular covariant value of the condensate derived in [6] in the confining vacuum:

$$
\text { Confining: } \quad\left\langle\operatorname{Tr} \Phi^{2}\right\rangle=-\frac{N}{12}\left(E_{2}(\tau / N)-N E_{2}(\tau)\right) .
$$


The expression for the condensate in the Higgs vacuum derived in [6] is

$$
\text { Higgs: } \quad\left\langle\operatorname{Tr} \Phi^{2}\right\rangle=-\frac{N^{2}}{12}\left(N E_{2}(\tau N)-E_{2}(\tau)\right) .
$$

As explained above, the expressions (3.5) and (3.6) are related by

$$
\left.\left\langle\operatorname{Tr} \Phi^{2}\right\rangle\right|_{\text {Higgs }}=\tau^{-2}\left(\left.\left\langle\operatorname{Tr} \Phi^{2}\right\rangle\right|_{\text {Conf }}\right)_{\tau \rightarrow-1 / \tau} .
$$

In order to see this one uses the modular transformation property of the Eisenstein series (A.14).

Now consider the $\Phi^{4}$ deformation. In this case the sum (3.2) gives

$$
\begin{aligned}
\left\langle\operatorname{Tr} \Phi^{4}\right\rangle_{\text {int }}= & \frac{N}{720}\left(10 E_{2}(\tau / N)^{2}-E_{4}(\tau / N)\right) \\
& +\frac{1}{6}\left((2 N-3) E_{2}(\tau / 2)-(2 N-6) E_{2}(\tau)\right)\left\langle\operatorname{Tr} \Phi^{2}\right\rangle_{\text {int }} \\
& +\frac{N}{720}\left(5 N(N-1) E_{2}(\tau / 2)^{2}-10 N(N-2) E_{2}(\tau)^{2}\right. \\
& \left.-(N-1)(N-3)^{2} E_{4}(\tau / 2)+N^{2}(N-2) E_{4}(\tau)\right) .
\end{aligned}
$$

In the large- $N$ limit one can easily see that (3.8) goes over to (2.33). In addition it vanishes for $N=2$ as remarked above. Notice that the difference between $\left\langle\operatorname{Tr} \Phi^{4}\right\rangle_{\mathrm{DV}}$ and $\left\langle\operatorname{Tr} \Phi^{4}\right\rangle_{\text {int }}$ is again by a vacuum independent, i.e. $\tau / N$-independent, admixture of $\operatorname{Tr} \Phi^{2}$ and the identity operator. Once again there is a natural modular covariant definition of the condensate which can be established by investigating the integrable system and establishing a relation between $\left\langle\operatorname{Tr} \Phi^{4}\right\rangle$ and $\left\langle\operatorname{Tr} \Phi^{4}\right\rangle_{\mathrm{int}}$. Using (3.8) one eventually finds

$$
\begin{aligned}
\left\langle\operatorname{Tr} \Phi^{4}\right\rangle=\left\langle\operatorname{Tr} \Phi^{4}\right\rangle_{\mathrm{DV}} & +\frac{1}{12}\left(6-N E_{2}(\tau)\right)\left\langle\operatorname{Tr} \Phi^{2}\right\rangle_{\mathrm{DV}} \\
& +\frac{N}{720}\left(N^{2} E_{4}(\tau)-5 N^{2} E_{2}(\tau)^{2}-35 N E_{2}(\tau)+9\right) .
\end{aligned}
$$

The result (2.33), then gives the value of the condensate in the confining vacuum:

$$
\text { Confining: } \quad \begin{aligned}
\left\langle\operatorname{Tr} \Phi^{4}\right\rangle & =\frac{N}{720}\left(10 E_{2}(\tau / N)^{2}-E_{4}(\tau / N)\right. \\
& \left.-5 N^{2} E_{2}(\tau)^{2}+N^{2} E_{4}(\tau)-5 N E_{2}(\tau) E_{2}(\tau / N)\right) .
\end{aligned}
$$

The value in the Higgs vacuum follows by taking $\tau \rightarrow-1 / \tau$ and multiplication by $\tau^{-4}$ yielding

$$
\text { Higgs: } \quad \begin{aligned}
\left\langle\operatorname{Tr} \Phi^{4}\right\rangle & =\frac{N}{720}\left(10 N^{4} E_{2}(\tau N)^{2}-N^{4} E_{4}(\tau N)\right. \\
& \left.-5 N^{2} E_{2}(\tau)^{2}+N^{2} E_{4}(\tau)-5 N^{3} E_{2}(\tau) E_{2}(\tau N)\right) .
\end{aligned}
$$




\section{Perturbation of the Leigh-Strassler deformation}

We now turn to an application of the techniques of Dijkgraaf and Vafa to a very different class of deformations of the $U(N) \mathcal{N}=4$ theory. We consider the $\mathcal{N}=4$ theory perturbed both by a certain exactly marginal operator (the Leigh-Strassler deformation [18]) and certain mass terms. (The authors of [4] have pointed out that such deformations can accessed via known matrix models.) The Leigh-Strassler deformations of the $\mathcal{N}=4$ theory lead to a 3 -dimensional fixed manifold of $\mathcal{N}=1$ theories, via special trilinear perturbations of the $\mathcal{N}=4$ superpotential. Among these marginal deformations, of particular interest is the so-called " $q$-deformation" of the $\mathcal{N}=4$ superpotential,

$$
W=\operatorname{Tr}\left(i \Phi\left[\Phi^{+}, \Phi^{-}\right]_{\beta}\right) \equiv \operatorname{Tr}\left(i \Phi\left[\Phi^{+} \Phi^{-} e^{i \beta / 2}-\Phi^{-} \Phi^{+} e^{-i \beta / 2}\right]\right) .
$$

We consider a special perturbation of this theory via the superpotential

$$
W=\operatorname{Tr}\left(i \Phi\left[\Phi^{+}, \Phi^{-}\right]_{\beta}+\Phi^{+} \Phi^{-}+\Phi^{2}\right)
$$

We choose this particular form for the mass perturbation as the corresponding large$N$ matrix model can be solved [19] around the trivial classical solution $\Phi=\Phi^{ \pm}=0$. This trivial solution corresponds of course to the confining vacuum of the $\mathcal{N}=1$ gauge theory.

As demonstrated in [19], expanding around the trivial vacuum the fields $\Phi^{ \pm}$ can be integrated out and the corresponding one-matrix model can be solved in the large- $N$ limit by going to an eigenvalue basis and a change of variables. We simply quote this change of variables here, without further explanation. In particular the eigenvalues $\lambda_{i}$ of $\Phi$ are redefined in terms of new variables $\delta_{i}$

$$
\lambda_{i}=-e^{\delta_{i}}+\frac{1}{2 \sin (\beta / 2)}
$$

The eigenvalues experience a force that causes them to condense and expand out into a cut in the large- $N$ limit. The complex $z$-plane in which the $\delta_{i}$ 's live turns out to be a cylinder with two cuts and gluing conditions on the two cuts implied by the saddle point equation for the function $G(z)$.

\subsection{The $\tilde{\tau}$ torus}

The key point for us is that large- $N$ matrix model solution once again leads to the emergence of a torus. The z-plane being a two-cut cylinder with certain gluing 
conditions, there is a natural map to a torus $E_{\tilde{\tau}}$ with 2 marked points. As before, we think of this torus as the complex $u$-plane modded out by lattice translations. The map $z(u)$ and $G(z(u))$ can be determined precisely using in particular the asymptotic behaviour of $G(z(u))$. We refer the reader to [19] for the details of these asymptotic properties. Using these properties we are able to determine $G(z(u))$ and the map $z(u)$ in the elliptic parametrization as

$$
G(z(u))=\frac{\omega_{1}^{2}}{\pi^{2}} \frac{\cos (\beta / 2)}{2 \sin ^{3}(\beta / 2)} \frac{\theta_{1}(\beta / 2)^{2}}{\theta_{1}^{\prime}(\beta / 2)^{2}}\left[\wp\left(u+\omega_{1} \beta / 2 \pi\right)-\wp\left(\omega_{1} \beta / \pi\right)\right]
$$

and

$$
\exp z(u)=\frac{1}{2} \cot (\beta / 2) \frac{\theta_{1}^{\prime}(0)}{\theta_{1}^{\prime}\left(\frac{\beta}{2}\right)} \frac{\theta_{1}\left(\pi u / 2 \omega_{1}-\beta / 4\right)}{\theta_{1}\left(\pi u / 2 \omega_{1}+\beta / 4\right)} .
$$

Note that $G(z(u))$ is elliptic while $z(u)$ is periodic only along the $A$-cycle while $B$-cycle shifts lead to shifts in $z(u)$,

$$
z\left(u+2 \omega_{1}\right)=z(u) ; \quad z\left(u+2 \omega_{2}\right)=z(u)+i \beta .
$$

This encodes the gluing conditions implied by the saddle point equation satisfied by $G(z)$. Note that the points $u= \pm \beta \omega_{1} / 2 \pi$ are the two marked points on the $\tilde{\tau}$-torus and they map to the points $z=\mp \infty$. Note also that in the $\beta \rightarrow 0$ limit $z(u)$ and $G(z(u))$ reduce to Eqs.(2.14) and (2.15) after a simple rescaling of $z(u)$ by $\beta$.

\subsection{The effective superpotential}

We can now use the Dijkgraaf-Vafa prescription to compute the effective superpotential for the relevant deformation of the Leigh Strassler theory. We have to compute the integrals of the one-form $G(z) d z$ over the $A$ and $B$ cycles of the $\tilde{\tau}$-torus. The integrals are surprisingly simple and we find

$\Pi_{A}=-i \int_{A} G(z(u)) z^{\prime}(u) d u=\frac{d h(\tilde{\tau})}{d \tilde{\tau}} ; \quad \Pi_{B}=-i \int_{B} G(z(u)) z^{\prime}(u) d u=\tilde{\tau} \frac{d h(\tilde{\tau})}{d \tilde{\tau}}-h(\tilde{\tau})$

where

$$
h(\tilde{\tau})=\frac{\cos (\beta / 2)}{4 \sin ^{3}(\beta / 2)} \frac{\theta_{1}(\beta / 2 \mid \tilde{\tau})}{\theta_{1}^{\prime}(\beta / 2 \mid \tilde{\tau})} .
$$

Thus we find the same structure as before for $S$ and $\partial \mathcal{F}_{0} / \partial S$ which guarantees that

$$
d W_{\mathrm{eff}} / d S=0 \Longrightarrow \tilde{\tau}=\tau / N
$$

and

$$
W_{\text {eff }}=-N h=-N \frac{\cos (\beta / 2)}{4 \sin ^{3}(\beta / 2)} \frac{\theta_{1}(\beta / 2 \mid \tau / N)}{\theta_{1}^{\prime}(\beta / 2 \mid \tau / N)}
$$


up to an additive constant. A quick check confirms that as $\beta \rightarrow 0$ this result reproduces the superpotential for $\mathcal{N}=1^{*}$ theory. ${ }^{5}$

\subsection{QFT eigenvalues and condensates}

We recall that in the deformations considered in Section 2, the map from the $A$-cycle of the the torus $E_{\tilde{\tau}}$ to the $z$-plane turned out to yield the field theory eigenvalues distributed uniformly on this cycle of $E_{\tilde{\tau}}$. We can ask if a similar picture emerges for perturbations of the Leigh-Strassler theory. We first note that we need to take into account the field redefinition Eq.(4.3) and so we need to consider the function $-\exp z(u)+1 /(2 \sin (\beta / 2))$ evaluated along the $A$-cycle of the torus, and we find that the relevant function (after a shift of $z$ by $i \beta / 2$ ) is

$$
\lambda(x)=-\frac{1}{2} \cot \left(\frac{\beta}{2}\right) \frac{\theta_{1}^{\prime}(0)}{\theta_{1}^{\prime}(\beta / 2)} \frac{\theta_{3}(\pi x-\beta / 4)}{\theta_{3}(\pi x+\beta / 4)}+\frac{1}{2 \sin (\beta / 2)} ; \quad x \in\left[-\frac{1}{2}, \frac{1}{2}\right] .
$$

It is quite remarkable that for the quadratic deformation considered in this section we find that indeed,

$$
W_{\text {eff }}=N \int_{-1 / 2}^{1 / 2} \lambda^{2}(x) d x=-N \frac{\cos (\beta / 2)}{4 \sin ^{3}(\beta / 2)} \frac{\theta_{1}(\beta / 2 \mid \tau / N)}{\theta_{1}^{\prime}(\beta / 2 \mid \tau / N)}+\frac{N}{4 \sin ^{2}(\beta / 2)}
$$

Although we don't have a general proof, the above leads us to believe that $\lambda(x)$ indeed yields the eigenvalues of the adjoint scalar in the confining vacuum of the field theory, distributed uniformly over the interval $\left[-\frac{1}{2}, \frac{1}{2}\right]$. In other words, replacing $x$ by a discrete index as in the previous section would precisely yield the eigenvalues of the adjoint scalar in the confining vacuum of the finite $N$ field theory. We further conjecture that our conclusions in Section 3 on the relation between the field theory quantities at large- $N$ and finite $N$ should also hold for the deformations of the LeighStrassler theories. Note also that our earlier comments on the distinction between field theory eigenvalue densities and matrix model eigenvalue densities apply in this case as well.

\section{A potential test involving S-duality:}

Although not completely understood in the context of this class of theories, one expects the Higgs and confining phases of these theories to be related by $S$ -

\footnotetext{
${ }^{5}$ Actually there is a $\tau$-independent additive constant which diverges in the $\beta \rightarrow 0$ limit which is a consequence of the linear shift of variables introduced in [19]. Strictly speaking, the resultant shift in the matrix model action $N /\left(4 \sin ^{2}(\beta / 2)\right)$ needs to be reintroduced into $W_{\text {eff }}$ which would then get rid of the offending additive term in the $\beta \rightarrow 0$ limit.
} 
duality i.e. $\tau \rightarrow-1 / \tau$. A possible non-trivial test of our results for the superpotential and eigenvalue distributions in the confining vacuum is a comparison with classical eigenvalues in the Higgs vacuum after performing $S$-duality on the confining vacuum and taking the semiclassical limit. It is not difficult to see that the

classical eigenvalues in the Higgs vacuum are distributed on a circle with $\tilde{\lambda}_{j} \sim$ $\exp (-i(N+1-2 j) \beta / 2) / \cos (N \beta / 2) ; j=1, \ldots N$. After an $S$-duality transformation, and redefining $\beta^{\prime}=\tau \beta$, in the semiclassical $\tau \rightarrow i \infty$ limit our result Eq.(4.11) also yields a uniform circular distribution of eigenvalues $\lambda(x) \sim \exp \left(-i \beta^{\prime} N x\right) / \cos (\beta N / 2)$; $x \in[-1 / 2,1 / 2]$. This gives a hint of how $S$-duality should act on the Leigh-Strassler deformation parameter. Indeed the Leigh-Strassler family of $\mathcal{N}=1 \mathrm{CFT}$ are not self-dual under $S L(2, \mathbb{Z})[18]$. Our observations here could provide a starting point for further investigation into these issues.

\section{Conclusions}

In this article we have performed a test of the proposal of [1-3] for a large class of $\mathcal{N}=1$ preserving deformations of the $\mathcal{N}=4$ theories in their confining phase. We have shown that the Dijkgraaf-Vafa proposal translates into a rather simple recipe for extracting eigenvalues of the adjoint scalar $\Phi$ in the confining vacuum from the corresponding matrix model, thereby yielding all the relevant condensates including the superpotential in that vacuum. We find that the eigenvalues extracted using this recipe match precisely with the results of [8] wherein the relevant eigenvalues were obtained from a completely different approach involving the connection of $\mathcal{N}=1^{*}$ theories with integrable models. Although at first sight the matrix model recipe yields large- $N$ field theory results, we demonstrate through certain nontrivial examples that these large- $N$ results are simply related to their finite $N$ counterparts via vacuum independent field redefinitions or operator mixings. Thus the large- $N$ matrix model indeed computes the holomorphic observables of the field theory at finite $N$ in its confining vacuum.

We also compute the effective superpotential in the confining vacuum of a mass deformation of the Leigh-Strassler CFT. We demonstrate that for this class of deformations, the matrix model once again yield the field theory eigenvalues and condensates via a simple recipe. A potential test of our results in the confining vacuum involves utilising $S$-duality to obtain the eigenvalues in the Higgs vacuum in the classical limit. The latter can be computed independently and we find nontrivial agreement if we assume that the Leigh-Strassler deformation parameter transforms with modular weight one, under S-duality. 
An interesting question concerns whether the matrix model approach can capture the full vacuum structure of the $\mathcal{N}=1^{*}$ theory, including the Higgs vacuum. We answer this in the affirmative in a separate publication.

Acknowledgements We would like to thank R. Dijkgraaf and C. Vafa for extensive stimulating discussions. S.P.K. would like to acknowledge support from a PPARC Advanced Fellowship.

\section{AppendixA: Some Properties of Elliptic Functions}

In this appendix we provide some useful — but far from complete - details of elliptic functions and their near cousins. For definitions and a more complete treatment, we refer the reader to one of the textbooks, for example [14]. An elliptic function $f(u)$ is a function on the complex plane, periodic in two periods $2 \omega_{1}$ and $2 \omega_{2}$. We will define the lattice $\Gamma=2 \omega_{1} \mathbb{Z} \oplus 2 \omega_{2} \mathbb{Z}$ and define the basic period parallelogram as

$$
\mathcal{D}=\left\{u=2 \mu \omega_{1}+2 \nu \omega_{2}, 0 \leq \mu<1,0 \leq \nu<1\right\}
$$

The complex structure of the torus defined by identifying the edges of $\mathcal{D}$ is

$$
\tau=\omega_{2} / \omega_{1}
$$

and we also define

$$
q=e^{i \pi \tau}
$$

\section{A.1 The Weierstrass function}

The archetypal elliptic function is the Weierstrass $\wp(u)$ function. It is an even function which is analytic throughout $\mathcal{D}$, except at $u=0$ where it has a double pole:

$$
\begin{aligned}
& \wp(u)=\frac{1}{u^{2}}+\sum_{k=1}^{\infty} c_{k+1} u^{2 k}, \\
& c_{2}=\frac{g_{2}}{20}, \quad c_{3}=\frac{g_{3}}{28}, \quad c_{k}=\frac{3}{(2 k+1)(k-3)} \sum_{j=2}^{k-2} c_{j} c_{k-j} \quad k \geq 4 .
\end{aligned}
$$

The Weierstrass function satisfies the fundamental identity

$$
\left(\frac{d}{d u} \wp(u)\right)^{2}=4 \wp(u)^{3}-g_{2} \wp(u)-g_{3}
$$

which defines the Weierstrass invariants $g_{2,3}=g_{2,3}\left(\omega_{\ell}\right)$ associated to the torus. 


\section{A.2 The Weierstrass zeta function}

We are also interested in other functions which are only quasi-elliptic. First we have $\zeta(u)$. It is an odd function with the quasi-elliptic property:

$$
\zeta\left(u+2 \omega_{\ell}\right)=\zeta(u)+2 \zeta\left(\omega_{\ell}\right)
$$

Its derivative gives minus the Weierstrass function

$$
\wp(u)=-\zeta^{\prime}(u)
$$

It follows that $\zeta(u)$ has a simple pole at $u=0$. Useful identities are

$$
\begin{aligned}
& \omega_{2} \zeta\left(\omega_{1}\right)-\omega_{1} \zeta\left(\omega_{2}\right)=\frac{\pi i}{2}, \\
& \zeta\left(\omega_{1}+\omega_{2}\right)=\zeta\left(\omega_{1}\right)+\zeta\left(\omega_{2}\right), \\
& \wp\left(\omega_{1}+\omega_{2}\right)+\wp\left(\omega_{1}\right)+\wp\left(\omega_{2}\right)=0 .
\end{aligned}
$$

\section{A.3 The Jacobi theta functions}

We are also interested in the Theta functions $\theta_{i}(x \mid \tau)$, or $\theta_{i}(x, q), i=1,2,3,4$. They are also quasi-elliptic functions on $\mathcal{D}$ when $x=\pi u / 2 \omega_{1}$. Each of them satisfies the heat equation

$$
\pi i \frac{\partial^{2} \theta_{i}(x \mid \tau)}{\partial x^{2}}+4 \frac{\partial \theta_{i}(x \mid \tau)}{\partial \tau}=0
$$

They are related to the previous functions; for instance, two relations that we need are

$$
\zeta(u)-\frac{\zeta\left(\omega_{1}\right)}{\omega_{1}} u=\left.\frac{\pi}{2 \omega_{1}} \frac{\theta_{1}^{\prime}(x \mid \tau)}{\theta_{1}(x \mid \tau)}\right|_{x=\pi u / 2 \omega_{1}} \equiv-\frac{i \pi}{2 \omega_{1}}+\left.\frac{\pi}{2 \omega_{1}} \frac{\theta_{3}^{\prime}(x \mid \tau)}{\theta_{3}(x \mid \tau)}\right|_{x=\pi\left(u-\omega_{1}-\omega_{2}\right) / 2 w_{1}},
$$

where the derivative is with respect to $x$.

\section{A.4 The Eisenstein series}

Finally, we introduce the Eisenstein series and their relation to elliptic functions. There are a number of ways to define these series: see $[16]^{6}$; for instance

$$
E_{k}(\tau)=\frac{1}{2} \sum_{\substack{m, n \in \mathbb{Z} \\(m, n)=1}} \frac{1}{(m \tau+n)^{k}}
$$

\footnotetext{
${ }^{6}$ Beware, the definition of $q$ in that reference is the square of ours.
} 
where $(m, n)$ denotes the greatest common divisor. Each series has a $q$-expansion; for example

$$
\begin{aligned}
& E_{2}(\tau)=1-24 \sum_{n=1} \sigma_{1}(n) q^{2 n}, \\
& E_{4}(\tau)=1+240 \sum_{n=1} \sigma_{3}(n) q^{2 n} \\
& E_{6}(\tau)=1-504 \sum_{n=1} \sigma_{5}(n) q^{2 n}
\end{aligned}
$$

where $\sigma_{j}(n)$ is a sum over each positive integral divisor of $n$ raised to the $j^{\text {th }}$ power.

Useful identities are

$$
\begin{aligned}
& E_{2}^{\prime}=\frac{\pi i}{6}\left(E_{2}^{2}-E_{4}\right) \\
& E_{4}^{\prime}=\frac{2 \pi i}{3}\left(E_{4} E_{2}-E_{6}\right) \\
& E_{6}^{\prime}=\pi i\left(E_{6} E_{2}-E_{4}^{2}\right)
\end{aligned}
$$

where the derivatives are defined with respect to $\tau$. Under a $S$-duality transformation we have

$$
E_{2}(-1 / \tau)=\tau^{2} E_{2}(\tau)+\frac{6 \tau}{\pi i}, \quad E_{k}(-1 / \tau)=\tau^{k} E_{k}(\tau) \quad k \geq 4
$$

The relations of the Eisenstein series to the elliptic functions defined previously appears through the identities

$$
\zeta\left(w_{1}\right) w_{1}=\frac{\pi^{2}}{12} E_{2}(\tau), \quad g_{2}\left(2 w_{1}\right)^{4}=\frac{4 \pi^{4}}{3} E_{4}(\tau), \quad g_{3}\left(2 w_{1}\right)^{6}=\frac{8 \pi^{8}}{27} E_{6}(\tau) .
$$

\section{AppendixB: Stationary points of the Hamiltonians}

In this Appendix we show that the configuration of the ECM system considered in the text is an equilibrium point with respect to each of the Hamiltonians $H_{p}=\operatorname{Tr} L^{p}$. In each case Hamilton's equations read $\partial P_{j} / \partial t=-\partial H_{p} / \partial X_{j}$ and $\partial X_{j} / \partial t=\partial H_{p} / \partial P_{j}$. Thus we need to show that the right hand side of both these equations vanishes in the configuration of interest where $X_{j}=\left\langle X_{j}\right\rangle=2 \pi i j / N$ for $j=1,2 \ldots N$ and $P_{j}=\left\langle P_{j}\right\rangle=0$. (Note that here for simplicity we present the requisite proof for the equilibrium positions that correspond to the Higgs vacuum of the gauge theory. The proof for the confining vacuum can be obtained along the same lines by using the Lax matrix appropriate for that vacuum and equilibrium positions $X_{j}=\left\langle X_{j}\right\rangle=2 \pi i \tau j / N$ for $j=1,2 \ldots N$ and $P_{j}=\left\langle P_{j}\right\rangle=0$.) 
Importantly we must also remember to impose the constraint appropriate to the $S U(N)$ theory: $H_{1}=\sum_{j=1}^{N} P_{j}=0$. This is most easily accomplished by introducing a Lagrange multiplier $\mu$ and looking for stationary points of the modified Hamiltonians $\tilde{H}_{p}=H_{p}-\mu H_{1}$. Thus we must show that the equations,

$$
\frac{\partial H_{p}}{\partial X_{j}}=0 \quad \frac{\partial H_{p}}{\partial P_{j}}=\mu
$$

Can be solved in the equilibrium configuration. We will first demonstrate that,

$$
\left.\frac{\partial H_{p}}{\partial X_{j}}\right|_{\left\langle X_{j}\right\rangle,\left\langle P_{j}\right\rangle}=0
$$

The Hamiltonians of the integrable system can be expressed with the Lax matrix as

$$
H_{p}=\operatorname{Tr}\left[L^{p}\right]
$$

so we would like to prove that for the Calogero-Moser system

$$
\left.\frac{\partial H_{p}}{\partial X_{j}}\right|_{\left\langle X_{j}\right\rangle,\left\langle P_{j}\right\rangle}=\left.\operatorname{Tr}\left[L^{p-1} \frac{\partial L}{\partial X_{j}}\right]\right|_{\left\langle X_{j}\right\rangle,\left\langle P_{j}\right\rangle}=0
$$

The CM system is described with the Lax matrix

$$
L_{j k}=p_{j} \delta_{j k}+i\left(1-\delta_{j k}\right) x\left(X_{j}-X_{k}\right)
$$

where the function $x(u)$ is given explicitly in [8]. For the present purposes we only need two properties of $x(u)$. Firstly it has period $2 \pi i: x(u+2 \pi i)=x(u)$. Secondly it is an odd function: $x(-u)=-x(u)$. As above, the equilibrium positions of the integrable system are

$$
\left\langle X_{j}\right\rangle=\frac{2 i \pi j}{N}-\frac{i \pi(N-1)}{N} \quad j=1 \ldots N
$$

and $\left\langle P_{j}\right\rangle=0$ The key point is that the Lax matrix becomes a circulant when evaluated in this configuration. It is then easy to show that the unitary transformation which diagonalises the Lax matrix is,

$$
\begin{aligned}
L & =U^{\dagger} \Lambda U \\
\Lambda_{j k} & =\lambda_{j} \delta_{j k}
\end{aligned}
$$

with

$$
U_{j k}=\frac{1}{\sqrt{N}} e^{\frac{2 \pi i}{N}(j-1)(k-1)}
$$


The trace then can be written

$$
\left.\operatorname{Tr}\left[L^{p-1} \frac{\partial L}{\partial X_{j}}\right]\right|_{\left\langle X_{j}\right\rangle,\left\langle P_{j}\right\rangle}=\operatorname{Tr}\left[\left.\Lambda^{p-1} U \frac{\partial L}{\partial X_{j}}\right|_{\left\langle X_{j}\right\rangle,\left\langle P_{j}\right\rangle} U^{\dagger}\right]
$$

Substituting the expressions for the Lax matrix and for $U$ the explicit form of the trace is

$$
\operatorname{Tr}\left[\left.\Lambda^{p-1} U \frac{\partial L}{\partial X_{j}}\right|_{\left\langle X_{j}\right\rangle,\left\langle P_{j}\right\rangle} U^{\dagger}\right]=-\frac{2}{N} \sum_{k=1}^{N} \lambda_{k}^{p-1} \sum_{l=1, l \neq j}^{N} x^{\prime}\left(X_{j}-X_{l}\right) \sin \frac{2 \pi}{N}(k-1)(j-l)
$$

Using that $x^{\prime}(u)$ is an even function and is periodic in $\omega_{1}=2 \pi i$ we find the terms in the summation pairwise cancel. Thus the summation gives zero and we conclude

$$
\left.\frac{\partial H_{p}}{\partial X_{j}}\right|_{\left\langle X_{j}\right\rangle,\left\langle P_{j}\right\rangle}=0
$$

Similarly its easy to show that the second equation in (B.1) is solved by choosing $\mu=H_{p-1}$. This completes the proof.

\section{References}

[1] R. Dijkgraaf and C. Vafa, "Matrix models, topological strings, and supersymmetric gauge theories," [hep-th/0206255].

[2] R. Dijkgraaf and C. Vafa, "On geometry and matrix models," [hep-th/0207106].

[3] R. Dijkgraaf, Talk at "Strings 2002", Cambridge, [http://www.damtp.cam.ac.uk/strings02/avt/dijkgraaf/]

[4] R. Dijkgraaf and C. Vafa, "A perturbative window into non-perturbative physics," [hep-th/0208048]

[5] O. Aharony, N. Dorey and S. P. Kumar, JHEP 0006, 026 (2000) [hep-th/0006008].

[6] N. Dorey, JHEP 9907, 021 (1999) [hep-th/9906011].

[7] N. Dorey and S. P. Kumar, JHEP 0002, 006 (2000) [hep-th/0001103].

[8] N. Dorey and A. Sinkovics, JHEP 0207, 032 (2002) [hep-th/0205151].

[9] R. Donagi and E. Witten, Nucl. Phys. B 460, 299 (1996) [hep-th/9510101].

[10] A. Gorsky, I. Krichever, A. Marshakov, A. Mironov and A. Morozov, Phys. Lett. B 355, 466 (1995) [hep-th/9505035]. 
[11] E. J. Martinec and N. P. Warner, Nucl. Phys. B 459, 97 (1996) [hep-th/9509161]; E. J. Martinec, Phys. Lett. B 367, 91 (1996) [hep-th/9510204].

[12] N. Dorey, T. J. Hollowood and S. Prem Kumar, Nucl. Phys. B 624, 95 (2002) [hep-th/0108221].

[13] V. A. Kazakov, I. K. Kostov and N. A. Nekrasov, Nucl. Phys. B 557, 413 (1999) [hep-th/9810035].

[14] E. T. Whittaker, G. N. Watson, A course of Modern Analysis, 4th edn., Cambridge University Press, 1927.

[15] N. Dorey, V. V. Khoze and M. P. Mattis, Phys. Lett. B 396, 141 (1997) [hep-th/9612231].

[16] N. Koblitz, "Introduction to Elliptic Curves and Modular Forms". Springer-Verlag, 2nd Edition 1993.

[17] C. Vafa and E. Witten, Nucl. Phys. B 431, 3 (1994) [hep-th/9408074].

[18] R. G. Leigh and M. J. Strassler, Nucl. Phys. B 447, 95 (1995) [hep-th/9503121].

[19] I. K. Kostov, Nucl. Phys. B 575, 513 (2000) [hep-th/9911023]. 